\title{
Psicooncología
}

ISSN: $1696-7240$

http://dx.doi.org/10.5209/PSIC.61439

\section{Fase cualitativa del desarrollo de una Escala de Motivos a favor y en contra de la Reconstrucción Mamaria (EMRM)}

\author{
Melina Miaja Ávila, ${ }^{1, *}$ José Moral de la Rubia²; Cynthia Villarreal-Garza ${ }^{3}$
}

Recibido: 23 de noviembre de 2017 / Aceptado: 20 de agosto de 2018

Resumen: Objetivo: Aunque en la clínica e investigación del cáncer de mama se considera las razones para hacerse la reconstrucción mamaria, no existe una escala validada para su evaluación. El objetivo del estudio fue crear una escala para medir los motivos a favor y en contra de la reconstrucción mamaria. Método: A través de la técnica de las redes semánticas naturales, se obtuvieron los ítems de la escala, y a través de un juicio de expertos estos fueron modificados. La técnica de las redes semánticas se aplicó a 65 mujeres mexicanas con cáncer de mama. El grupo de expertos quedó formado por 12 especialistas en cáncer de mama. Resultados: El núcleo central de los motivos para hacerse la reconstrucción mamaria se compuso de 16 motivos, los cuales se pueden agrupar en estéticos/imagen corporal, funcionales, emocionales/psicológicos y de relación con los hijos y la pareja. El núcleo central de los motivos en contra de hacerse la reconstrucción mamaria se compuso de 11 motivos, los cuales se pueden agrupar en miedos, aceptación/comodidad con la situación presente, costos y salud. Desde estos contenidos se redactaron 16 ítems sobre motivos para hacerse la reconstrucción y 11 en contra. Esta escala de 27 ítems fue evaluada en adecuación y comprensibilidad por el grupo de 12 expertos. Se conservaron los 27 ítems, pero siete ítems fueron modificados. Finalmente, se añadieron dos ítems sobre la falta de información señalada en estudios en México. Conclusiones: Se sugiere estudiar la confiabilidad, validez y distribución de la escala de 29 ítems.

Palabras clave: cáncer de mama; reconstrucción mamaria; mastectomía; motivos; redes semánticas naturales.

\section{[en] Qualitative phase of the development of a Scale of Motives for and against Breast Reconstruction (SMBR)}

\footnotetext{
Abstract: Objective: Although the reasons for breast reconstruction are considered in clinics and research on breast cancer, there is not any validated scale for its evaluation. The objective of the study was to create a scale to measure the reasons for and against breast reconstruction. Method: Through the technique of natural semantic networks, the items of the scale were obtained, and through an expert judgment these were modified. The technique of semantic networks was applied to 65 Mexican women with breast cancer. The group of experts was formed by 12 specialists in breast cancer.

1 Melina Miaja Ávila. Tecnológico de Monterrey, Centro de Cáncer de Mama. Monterrey. México.

E-mail: miajaam@live.com.mx

2 José Moral de la Rubia. Facultad de Psicología, Universidad Autónoma de Nuevo León. Nuevo León. México

E-mail: josemoral@hotmail.com

3 Cynthia Villarreal-Garza. Tecnológico de Monterrey, Centro de Cáncer de Mama. Monterrey. México

E-mail: cynthiavg@gmail.com

* Dirección de correspondencia: Melina Miaja Ávila. Tecnológico de Monterrey, Centro de Cáncer de Mama.

Batallón de San Patricio, 112 Real de San Agustín. C.P. 66278, San Pedro Garza García, N.L., México.

E-mail: miajaam@live.com.mx
} 
Results: The central core of the reasons in favor of breast reconstruction was composed of 16 reasons, which can be grouped into esthetic/body image, functional, emotional/psychological and relationship with children and the couple. The core of the motives against breast reconstruction was composed of 11 motives, which can be grouped into fears, acceptance/comfort with the present situation, costs and health. Based on these contents, 16 items were written about reasons for reconstruction and 11 against reconstruction. This 27 -item scale was evaluated in adequacy and comprehensibility by the group of 12 experts. The 27 items were conserved, but seven items were modified. Finally, two items on the lack of information were added, which was marked in Mexican studies. Conclusions: It is suggested to study the reliability, validity and distribution of the 29 item scale.

Keywords: Breast cancer; breast reconstruction; mastectomy; motives; natural semantic networks.

Sumario. 1. Introducción 2. Método 3. Resultados 3.1. Descripción de la muestra 3.2. Prueba de asociación libre con las pacientes y redacción de los ítems de la escala 3.2.1. Razones a favor de la reconstrucción mamaria 3.2.2. Razones contra la reconstrucción mamaria 3.3. Validez de contenido de la escala a través del juicio de expertos 4. Discusión 5. Agradecimientos 6. Referencias bibliográficas Anexo.

Cómo citar: Miaja Ávila1 M, Moral de la Rubia J, Villarreal-Garza C. Fase cualitativa del desarrollo de una Escala de Motivos a favor y en contra de la Reconstrucción Mamaria (EMRM). Psicooncología 2018;15:327-344. doi: 10.5209/PSIC.61439.

\section{Introducción}

Previo al consenso de los Institutos Nacionales de Salud ${ }^{(1)}$, la mastectomía era el estándar de atención para las mujeres con cáncer de mama en los Estados Unidos. A partir de dicho consenso, la cirugía conservadora de mama junto con la radioterapia han sido el método quirúrgico más común para atender a pacientes en etapas tempranas, disminuyendo las mastectomías del $40 \%$ en el 2000 al $37 \%$ en el $2006^{(2)}$.

No obstante, en países con ingresos intermedios, la mastectomía sigue predominando debido a las insuficiencias en infraestructura y recursos humanos para proporcionar la radioterapia y debido al alto número de casos que se detectan en etapas avanzadas ${ }^{(3)}$. Precisamente, en el Instituto Nacional de Cancerología en México en 2008, solo el 15\% de las pacientes habían sido intervenidas con cirugía conservadora debido al alto número de casos detectados en etapas avanzadas, alrededor del $80 \%{ }^{(3)}$.

En México, es común que la mayoría de las pacientes (cerca de 90\%) se palpen por sí mismas la presencia del algún síntoma o signo de cáncer mamario, esto se debe a que la mayor parte de las mujeres son diagnosticadas en etapas avanzadas ${ }^{(3,4)}$. Siendo solo el $10 \%$ de las pacientes diagnosticadas en estadio I, el cual se caracteriza por un tumor con un tamaño máximo de $2 \mathrm{~cm}$ y si hay células cancerígenas en los ganglios linfáticos próximos alcanzan un máximo de $2 \mathrm{~mm}^{(4,5)}$.

A su vez, las tasas de mastectomía profiláctica han aumentado ${ }^{(2,6)}$ debido al miedo a la recurrencia y al incremento del uso de pruebas para detectar mutaciones de los genes BRCA1 y BRCA2 $2^{(7)}$.

A pesar de que se ha demostrado que la reconstrucción de mama brinda beneficios psicológicos a la mayoría de las pacientes que fueron intervenidas con mastectomía ${ }^{(8)}$, la cifra de mujeres que son intervenidas con este procedimiento continúa siendo baja ${ }^{(9-14)}$.

Manne et al. ${ }^{(15)}$ y Keith et al. ${ }^{(16)}$ han elaboraron cuestionarios que miden diversos motivos para realizarse o no la reconstrucción mamaria. Sin embargo, 
ambos estudios no han determinado las propiedades métricas de confiabilidad y validez. Manne et al. ${ }^{(15)}$ desarrollaron un instrumento que mide 17 motivos para elegir la reconstrucción mamaria y 8 motivos para no elegirla, y reportaron la media y desviación estándar de sus 25 ítems. Los principales motivos para elegir la reconstrucción mamaria fueron el deseo de tener los pechos del mismo tamaño $(\mathrm{M}=4,38 ; \mathrm{DT}=0,78)$, querer despertar después de la mastectomía con los pechos en su lugar $(\mathrm{M}=4,13$; DT $=0,88)$, para no sentir la molestia de mirarse al espejo y ver una cicatriz sin mama $(M=4,02 ; \mathrm{DT}=1,18)$, y para sentirse completa de nuevo $(\mathrm{M}=3,98 ; \mathrm{DT}=0,93)$. Por su parte, los principales motivos para no elegir la reconstrucción mamaria estaban relacionados con los riesgos médicos: no querer tener más cirugías de las que se necesitan $(\mathrm{M}=3,93$; $\mathrm{DT}=1,17)$, preocupación por los riesgos y posibles complicaciones de la reconstrucción mamaria $(\mathrm{M}=3,76$; DT $=0,96)$, y preocupación por los problemas a largo plazo que pueden ocurrir después de la reconstrucción mamaria $(\mathrm{M}=3,27 \text {; } \mathrm{DT}=1,04)^{(15)}$.

Por otra parte, Keith et al. ${ }^{(16)}$ reportaron los porcentajes de las cinco opciones de respuesta en las preguntas del cuestionario, separando a los grupos de mujeres que querían o no realizarse la reconstrucción. Además, estimaron un modelo de regresión logística en el cual menor edad y presencia de síntomas de depresión predijeron desear realizarse la reconstrucción mamaria ${ }^{(16)}$. Ananian et al. ${ }^{(17)}$ usaron preguntas dicotómicas para estudiar los determinantes de la elección de la reconstrucción mamaria tras mastectomía. Hallaron que el consejo médico $(\mathrm{OR}=$ 13,45; IC 95\%: [2,66-67,88]), una mejor percepción de la propia imagen corporal $(\mathrm{OR}=10,55$; IC 95\%: $[2,75-40,51])$, hablar de la cirugía con la pareja $(\mathrm{OR}=3,59$; IC 95\%: $[1,01-2,82])$ y menor temor a la cirugía $(\mathrm{OR}=0,69$; IC 95\%: $[0,56-0,84])$ predijeron el realizarse la reconstrucción mamaria. No obstante, estas preguntas no se contemplaron como una escala ${ }^{(17)}$. También existen investigaciones cualitativas enfocadas al estudio de estas actitudes y motivos, realizadas con muestras reducidas y preguntas abiertas, como la de $\mathrm{Fu}$, Milee, Chen y $\mathrm{Hsu}^{(18)}$. Estos investigadores en una muestra de 35 mujeres inmigrantes asiáticas en Estados Unidos hallaron que la funcionalidad, la edad, la percepción sobre la cirugía plástica, el miedo a los implantes, las barreras en las comunicaciones con los doctores y la falta de información fueron las categorías temáticas presentes en el discurso de las pacientes $^{(18)}$.

Aunque en la clínica e investigación del cáncer de mama se considera las razones para hacerse la reconstrucción mamaria, no existe una escala validada para su evaluación tan sólo propuestas de preguntas dicotómicas u ordinales usadas para desarrollar modelos de regresión logística. La creación de un instrumento que permita medir los motivos a favor y en contra de la reconstrucción mamaria no sólo permitiría identificar y estudiar los factores que influyen en la toma de decisiones de dicho procedimiento quirúrgico, sino que también permitiría evaluar estas motivaciones en la consulta para la toma de decisiones clínicas y la orientación del servicio. A su vez, posibilitaría el desarrollo de modelos estructurales acerca de los factores que subyacen a estas motivaciones.

El propósito de esta investigación es crear una escala para medir los motivos a favor y en contra de la reconstrucción mamaria desde un enfoque empírico, considerando los argumentos expresados por las pacientes y la valoración por expertos de los ítems redactados a partir de estos argumentos. Una vez se cuente con la escala, en estudios posteriores, se pretende elaborar una teoría estructural 
de los mismos y baremos interpretativos de la escala y sus factores. Este estudio parte del registro de los motivos referidos por las pacientes, usando la técnica de las redes semánticas naturales para su análisis. Desde los contenidos registrados, se redactan los ítems de la escala. Se prosigue con la valoración por un grupo de expertos de la adecuación y comprensibilidad de los ítems creados. Para finalmente obtener una escala apta para su estudio cuantitativo. Por tanto, se trata de la fase cualitativa del desarrollo de la escala. Los objetivos de esta investigación son: 1) describir y analizar los contenidos asociados a las preguntas estímulo sobre los motivos para hacerse o no la reconstrucción mamaria por un grupo de mujeres mastectomizadas, 2) redactar ítems a partir de estos contenidos, 3) evaluar el grado de adecuación y comprensibilidad de los ítems por un grupo de expertos, y 4) seleccionar y modificar los ítems para la propuesta final de la escala.

\section{Método}

Se realizó un estudio cualitativo, exploratorio y descriptivo. Se usó un diseño transversal y un muestreo no probabilístico. Por una parte, se colectó una muestral incidental de mujeres con diagnóstico de cáncer de mama y con mastectomía (pacientes). Por otra parte, se trabajó con una muestra intencional de expertos en diagnóstico y tratamiento de cáncer de mama.

Los criterios de inclusión para la muestra de pacientes fueron: 1) ser mujer, 2) diagnóstico de cáncer de mama, 3 ) mastectomía, y 4) proporcionar el consentimiento informado. Criterios de exclusión: 1) mastectomía profiláctica y 2) cirugía conservadora de mama. Criterios de eliminación: 1) dar sólo un motivo en la prueba de asociación libre.

Los criterios para escoger a los expertos fueron: 1) carrera profesional en ciencias de la salud, 2) al menos grado de especialidad o maestría, 3) experiencia en tratamiento de pacientes con cáncer de mama de al menos cinco años, y 4) experiencia en investigación.

La muestra de pacientes quedó integrada por 65 mujeres con mastectomía unilateral atendidas en el Centro de Cáncer de Mama del hospital San José, Monterrey, Nuevo León, México. El grupo de expertos quedó constituido por 12 profesionales: seis cirujanos, tres oncólogos, una patóloga, un radioterapeuta y una nutrióloga (siete hombres y cinco mujeres). La muestra de pacientes se recolectó de junio a septiembre de 2017. La evaluación por los expertos se realizó en septiembre de 2017.

A las pacientes se les aplicó una prueba de asociación libre por escrito. Se formuló una primera pregunta cerrada: ¿le gustaría realizarse la reconstrucción mamaria? (Sí/No). En función de la respuesta dada se preguntaba: "si su deseo es reconstruirse, mencione cinco razones por las cuales se reconstruiría la mama" o "si no está interesada en reconstruirse mencione cinco razones por las cuales no se haría la reconstrucción de mama”. A continuación, se pedía: “coloque en orden de importancia cada una de las razones que escribió. Para esto escriba el número 1 junto al motivo que crea más importante y así consecutivamente hasta enumerar las cinco razones". Las aplicaciones fueron al finalizar sus consultas de psicología, oncología o cirugía. El profesional entregaba la hoja en la que las preguntas y espacios de respuesta estaban impresos. Las pacientes respondían sobre la mesa 
del consultorio. Tardaban en responder de dos a cinco minutos y entregaban el cuestionario al profesional que permanecía en la consulta mientras respondían.

A los expertos se les entregó un cuestionario con los ítems redactados en el cuál tenía que evaluar los mismos en dos escalas. Una sobre la adecuación del contenido con tres opciones valorativas ("no es adecuado"; "sí, pero requiere cambios", "sí es adecuado") y otra sobre la claridad de la redacción del ítem con dos opciones valorativas ("sí", "no"). En el caso de señalarse que necesitaba cambios o falta de claridad, se dejaba un espacio para indicar cuáles y cómo se podría redactar mejor el ítem.

Se pidió el consentimiento informado expresado verbalmente a las pacientes. Primero se explicó en qué consistía la prueba, la finalidad del estudio y los responsables del mismo. A continuación, se pedía la participación voluntaria y sin remuneración. Se garantizaba la confidencialidad de los datos y se aclaraba que la participación o no en el estudio era totalmente independiente de su atención clínica. En caso de rehusarse a participar se preguntaba el motivo. Todas las pacientes a las que se les invitó dieron su consentimiento. El estudio fue aprobado por las Comisiones de Ética y de Investigación de la Escuela de Medicina del Tecnológico de Monterrey y de la Fundación Santos y de la Garza Evia I.B.P. (Hospital San José).

Para analizar la prueba de asociación libre se usó la técnica de las redes semánticas naturales ${ }^{(19)}$. Se contó el número de palabras asociadas (valor J), el cual proporciona información sobre la riqueza de la red semántica. Se calculó la frecuencia de cada palabra definidora (asociada a la frase estímulo) y su valor semántico (VM). Se dio un valor de 5 cuando la palabra era colocada en el primer orden de importancia entre las palabras asociadas, 4 si era puesta en segundo lugar, 3 en tercero, 2 en cuarto, y 1 en quinto. La suma de los valores correspondientes a las $\mathrm{k}$ veces en que fue asociada palabra definidora constituye su valor semántico (VM). Se ordenaron las palabras por su VM. Se elaboró una curva de sedimentación, poniendo en el eje horizontal las palabras ordenadas y en el eje vertical el VM. El punto de inflexión o pérdida de la pendiente en la curva proporcionó el criterio para seleccionar las palabras que conformaron el conjunto SAM (por sus siglas en inglés: Semantic Association Memory) o núcleo central de la representación. Fueron aquellas que quedaron por encima del punto de inflexión. Ordenadas por su VM, se calcularon las distancias de las palabras del conjunto SAM con respecto a la primera. Se dio un valor de 100 a la palabra con más VM, y se asignó la distancia a las demás palabras usando una regla de tres: VM de la palabra multiplicado por 100 y dividido por el VM de la primera palabra del conjunto SAM. Con estas distancias se realizó un gráfico de radial. Mayor apertura del radial refleja más diversidad en los significados que integran el núcleo central. Un cierre brusco indica un consenso rígido y monolítico ${ }^{(19)}$.

Desde los núcleos centrales de los motivos a favor y en contra de la reconstrucción mamaria se redactaron los ítems de la escala. Esta se entregó al grupo de expertos. Para analizar el juicio de los mismos, se calculó el porcentaje de las opciones de adecuación y comprensibilidad. El ítem fue retenido sin cambio cuando los porcentajes de adecuación y comprensibilidad eran mayores que $80 \%$. El ser señalado como inadecuado por más del $50 \%$ se definió como criterio de eliminación. En los demás casos se consideró las sugerencias de los jueces para mejorar la adecuación o comprensibilidad del ítem ${ }^{(20)}$. 


\section{Resultados}

\subsection{Descripción de la muestra}

La muestra clínica total quedó conformada por 65 mujeres con mastectomía unilateral. El 50,8\% de las participantes deseaban realizarse la reconstrucción mamaria y $49,2 \%$ no. La media de edad fue de 45,98 años (DT = 10,16). El 29,2\% de las mujeres fueron diagnosticadas en una etapa clínica IIB y la media del tiempo transcurrido desde la cirugía fue de 1,56 años $(\mathrm{DT}=1,02)$ (véase Tabla 1$)$.

Tabla 1. Variables sociodemográficas y clínicas

\begin{tabular}{cccc}
\hline Variables & Categorías & $\mathrm{n}(\%)$ & M [IC del 95\%] \\
\hline Edad & & & $45,98[43,7,48,50]$ \\
Tiempo desde la cirugía & & & $1,56[1,31,1,81]$ \\
Estadío & I & $2(3,1 \%)$ & \\
& IIA & $17(26,2 \%)$ & \\
& IIB & $19(29,2 \%)$ & \\
& IIIA & $16(24,6 \%)$ & \\
& IIIB & $6(9,2 \%)$ & \\
Lateralidad & IIIC & $3(4,6 \%)$ & \\
del tumor & IV & $2(3,1 \%)$ & \\
Desea hacerse & Derecha & $29(44,6 \%)$ & \\
reconstrucción mamaria & Izquierda & $36(55,4 \%)$ & \\
\hline
\end{tabular}

$\mathrm{n}=$ frecuencia absoluta simple, $\mathrm{M}$ = media aritmética, $\mathrm{IC}=$ intervalo de confianza. Fuente: elaborada por los autores.

$\mathrm{Al}$ dividir la muestra clínica en relación con su deseo o no de reconstruirse, la media del tiempo transcurrido desde la cirugía fue equivalente en ambas muestras $(\mathrm{t}[63]=0,727, \mathrm{p}=0,47)$. No obstante, fueron diferenciales en cuanto a la media de edad $(\mathrm{t}[63]=4,763, \mathrm{p}<0,01)$, siendo más jóvenes las mujeres que sí deseaban reconstruirse $(\mathrm{M}=40,88$ años, $\mathrm{DT}=7,47)$ que las que no desean reconstruirse $(\mathrm{M}=$ $51,25$ años, $\mathrm{DT}=9,95)$. A su vez, fueron diferenciales en relación a la etapa clínica en que fueron diagnosticadas $\left(Z_{U}=-3,185, p=0,001\right)$; las mujeres que no desean reconstruirse fueron diagnosticadas en una etapa más avanzada $(40,6 \%$ en etapa IIIA) que las mujeres que sí desean reconstruirse ( $42,4 \%$ en etapa IIB). 


\subsection{Prueba de asociación libre con las pacientes y redacción de los ítems de la escala}

\subsubsection{Razones a favor de la reconstrucción mamaria}

Ante la frase-estímulo "mencione cinco razones por las cuáles se haría la reconstrucción mamaria", el número de palabras definidoras fue 38 (valor J). Por el criterio del punto de pérdida de la pendiente en la curva de sedimentación del VM, el número de palabras definidoras del conjunto SAM o núcleo central fueron 15 (véase Figura 1).

Figura 1. Curva de sedimentación del valor semántico de las 41 palabras definidoras.

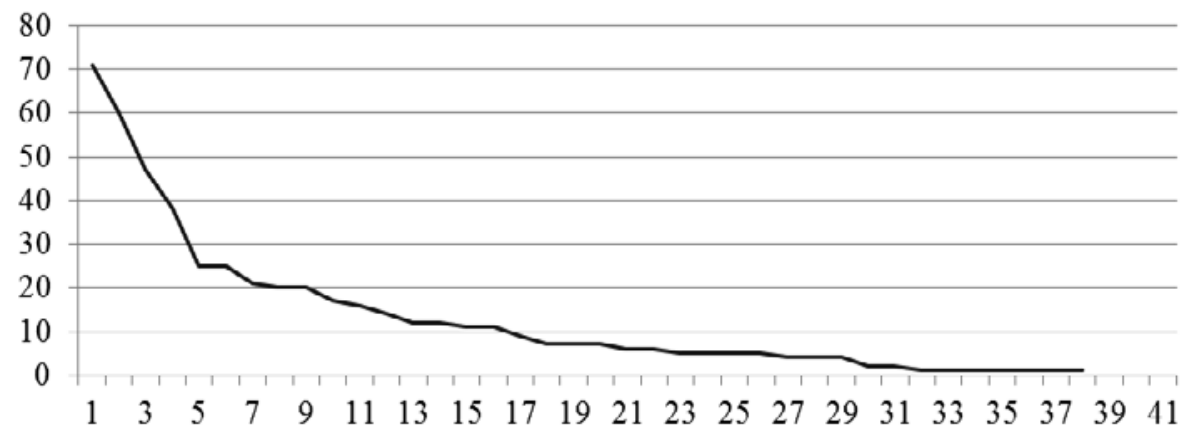

Fuente: elaborada por los autores.

Las 15 palabras-estímulos del conjunto SAM se pueden ver en la Tabla 2. Su perfil de radial mostró bastante apertura (véase Figura 2).

Tabla 2. Conjunto SAM

\begin{tabular}{ccccc}
\hline Orden & Palabra & FA & VM & FMG \\
\hline 1 & Sentirse bien & 17 & 71 & 0 \\
2 & Por estética & 20 & 60 & 3 \\
3 & Sentirse completa & 14 & 47 & 27 \\
4 & Mejorar autoestima & 10 & 38 & 45 \\
5 & Verse bien vestida & 10 & 25 & 53 \\
6 & Problemas prótesis externa & 9 & 25 & 56 \\
7 & Mejorar estado emocional & 7 & 21 & 65 \\
8 & Facilidad de elegir ropa & 6 & 20 & 69 \\
9 & Verse como estaba antes & 5 & 20 & 71 \\
10 & Sentirse más segura & 6 & 17 & 72 \\
11 & Simetría & 5 & 16 & 74 \\
12 & Sentirse más femenina & 4 & 14 & 74 \\
13 & Verse al espejo & 3 & 12 & 79 \\
14 & Por sus hijos & 3 & 12 & 83 \\
15 & Cerrar el ciclo & 6 & 11 & 83 \\
\hline
\end{tabular}

$\mathrm{FA}=$ frecuencia de aparición, $\mathrm{VM}=$ valor o peso semántico, $\mathrm{FMG}$ = distancia semántica a la palabra de mayor peso en el conjunto SAM o núcleo central. Fuente: elaborada por los autores. 
Figura 2. Diagrama de radial para el conjunto SAM basado en las distancias semánticas a la palabra de mayor peso.

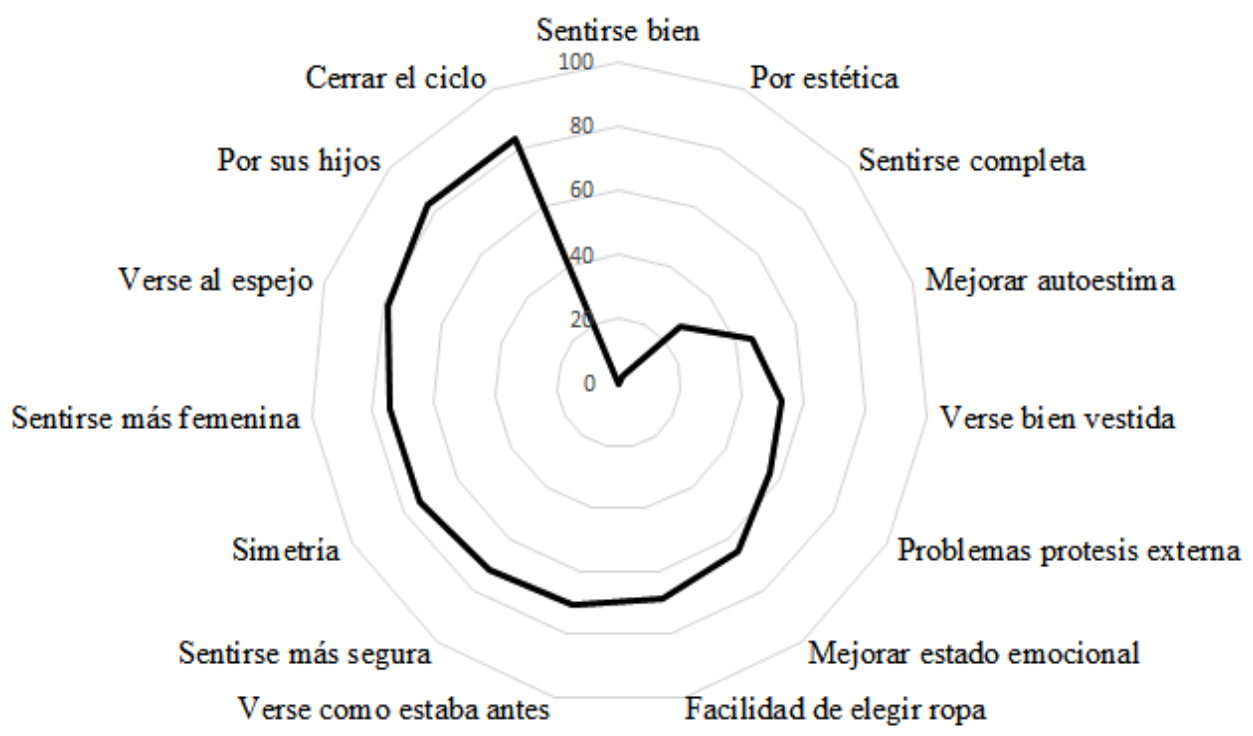

Fuente: elaborada por los autores.

\subsubsection{Razones contra la reconstrucción mamaria}

Ante la frase-estímulo "mencione cinco razones por lo cuáles no se haría la reconstrucción mamaria", el número de palabras definidoras fue 36 (valor J). Por el criterio del punto de pérdida de la pendiente en la curva de sedimentación del VM, el número de palabras definidoras del conjunto SAM o núcleo central fueron 11 (véase Figura 3).

Figura 3. Curva de sedimentación del valor semántico de las 36 palabras definidoras.

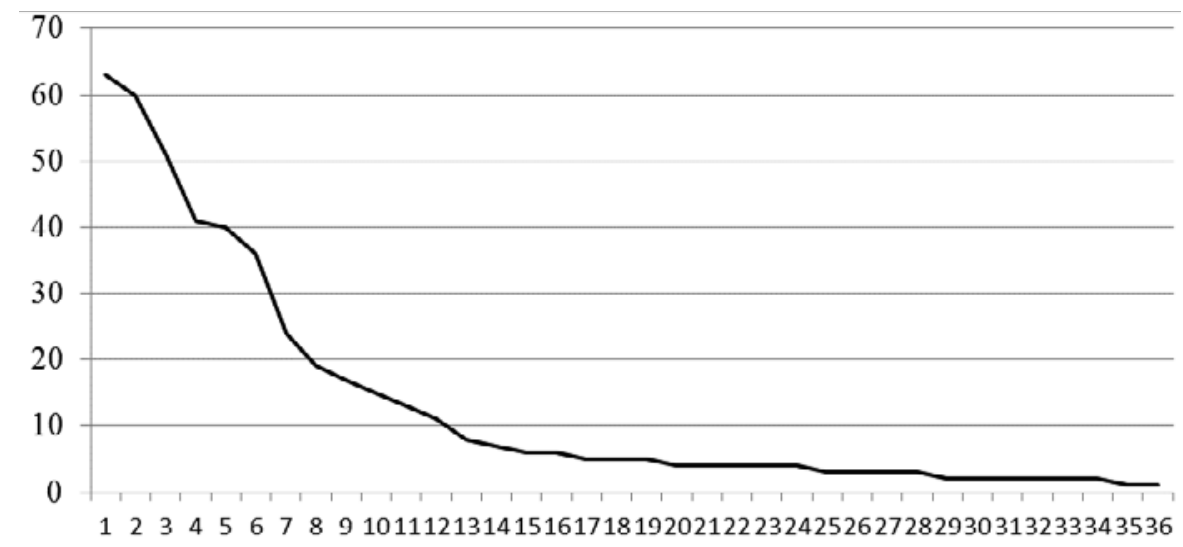

Fuente: elaborada por los autores. 
Las 11 palabras-estímulos del conjunto SAM se pueden ver en la Tabla 3. La Figura 4 muestra un perfil abierto en sus primeras cuatro palabras, pero con cierre abrupto en las últimas.

Tabla 3. Conjunto SAM

\begin{tabular}{ccccc}
\hline Orden & Palabra- estímulo & FA & VM & FMG \\
\hline 1 & Miedo a complicaciones en cirugía & 20 & 63 & 0 \\
2 & Por el costo/recurso económico & 16 & 60 & 5 \\
3 & No más procedimientos quirúrgicos/hartazgo & 19 & 51 & 19 \\
4 & Innecesario & 13 & 41 & 35 \\
5 & Aceptación corporal & 13 & 40 & 37 \\
6 & Edad avanzada & 11 & 36 & 43 \\
7 & Dificultad para realizar su vida diaria en recuperación & 6 & 24 & 62 \\
8 & Mayor seguridad/comodidad con prótesis externas & 7 & 19 & 70 \\
9 & Miedo al dolor/no tener dolor después de la cirugía & 4 & 17 & 73 \\
10 & Temor de recurrencia & 6 & 15 & 76 \\
11 & Sobrepeso & 3 & 13 & 79 \\
\hline
\end{tabular}

$\mathrm{FA}=$ frecuencia de aparición, $\mathrm{VM}$ = valor o peso semántico, $\mathrm{FMG}$ = distancia semántica a la palabra de mayor peso.

Figura 4. Diagrama de radial para el conjunto SAM basado en las distancias semánticas a la palabra de mayor peso.

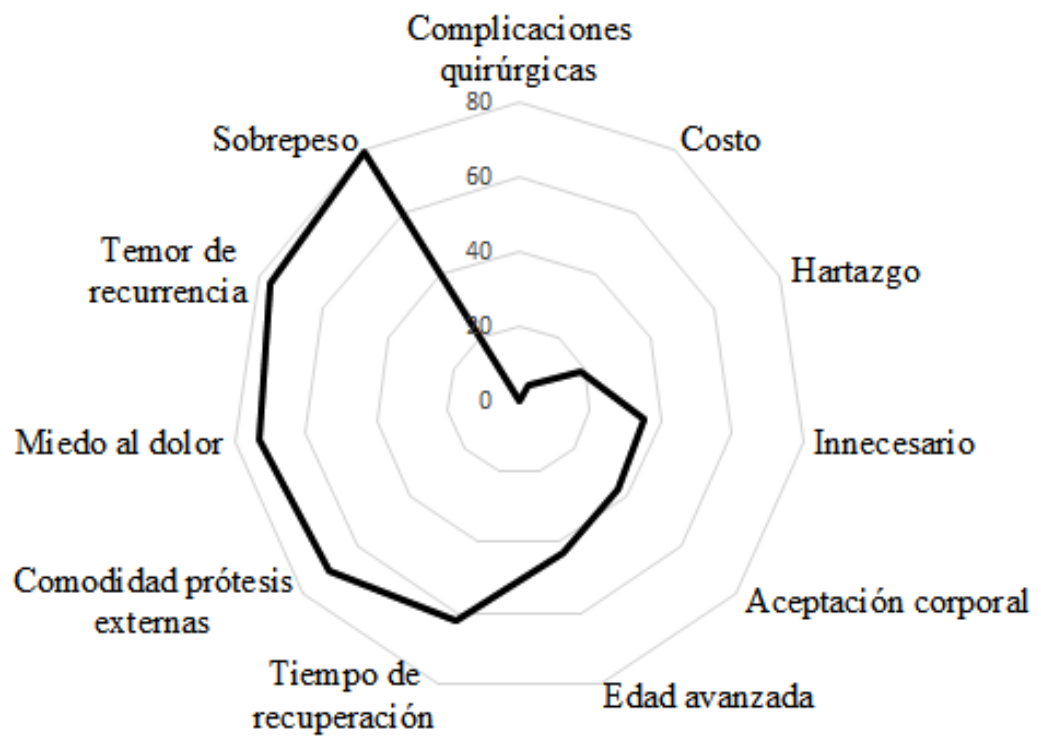

Fuente: elaborada por los autores. 
En la Tabla 4, se presenta la derivación de los ítems a partir de las palabras definidoras que constituyen ambos conjuntos SAM.

Tabla 4. Derivación de ítem a partir de la palabra definidora

\begin{tabular}{|c|c|c|}
\hline & Definidoras & Ítems redactados \\
\hline \multicolumn{3}{|c|}{ Motivos para hacerse la reconstrucción mamaria } \\
\hline 1 & Sentirse bien consigo misma & $\begin{array}{l}\text { Porque me siento mal conmigo misma des- } \\
\text { de la cirugía }\end{array}$ \\
\hline 2 & Por estética & $\begin{array}{l}\text { Porque me siento menos atractiva desde la } \\
\text { cirugía }\end{array}$ \\
\hline 3 & Sentirse completa como mujer & Porque me siento incompleta como mujer \\
\hline 4 & Mejorar autoestima & Para mejorar mi autoestima \\
\hline 5 & Verse bien vestida & $\begin{array}{l}\text { Porque no me gusta como se me ve ahora } \\
\text { la ropa }\end{array}$ \\
\hline 6 & $\begin{array}{l}\text { No tener problema con prótesis } \\
\text { externa }\end{array}$ & $\begin{array}{l}\text { Porque la prótesis externa me genera mu- } \\
\text { chos problemas }\end{array}$ \\
\hline 7 & Mejorar estado emocional & Para mejorar mi estado emocional \\
\hline 8 & Facilidad para elegir ropa y vestirse & $\begin{array}{l}\text { Porque la reconstrucción mamaria me daría } \\
\text { mayor libertad de vestir todo tipo de ropa }\end{array}$ \\
\hline 9 & Verse como estaba antes & Para verme como estaba antes de la cirugía \\
\hline 10 & Sentir más segura & Para sentirme más segura \\
\hline 11 & Simetría & Para verme simétrica de los pechos \\
\hline 12 & Sentirse más femenina & Para sentirme más femenina \\
\hline 13 & Verse al espejo desnuda & Para verme desnuda frente al espejo \\
\hline 14 & Por sus hijos & $\begin{array}{l}\text { Para que mis hijos no vean algo raro o dife- } \\
\text { rente en mis pechos }\end{array}$ \\
\hline 15 & Cerrar el ciclo del cáncer & $\begin{array}{l}\text { Porque la reconstrucción me permitiría ce- } \\
\text { rrar este capítulo en mi vida }\end{array}$ \\
\hline
\end{tabular}

\section{Motivos para no hacerse la reconstrucción mamaria}

1 Miedo a complicaciones en cirugía.

Porque tengo miedo de que se puedan presentar complicaciones en la reconstrucción mamaria

2 Por el costo/recurso económico.

Porque me preocupa el alto costo que puede llevar la reconstrucción mamaria

3 Hartazgo por tantos procedimientos Porque estoy harta de tantos procedimientos quirúrgicos 


\begin{tabular}{lll}
\hline \multicolumn{1}{c}{ Definidoras } & \multicolumn{1}{c}{ Ítems redactados } \\
\hline Motivos para no hacerse la reconstrucción mamaria
\end{tabular}

Fuente: elaborada por los autores.

Un motivo a favor de la reconstrucción mamaria fue "por el esposo". Cuatro de las 65 mujeres $(6,2 \%)$ lo mencionaron. Esta palabra definidora tuvo un valor semántico bajo ( $\mathrm{VM}=7$ en un rango de 71 a 1 con una media de 13,29), por lo que no quedó en el núcleo central. Si se pondera con un valor de 10 al contenido ordenado en primer lugar de representatividad y de cinco al último, siguiendo la ponderación usada con pruebas no acotadas a un máximo de palabras, entonces este motivo quedaría en el núcleo central y saldría el de "por los hijos". En las demás palabras definidoras que integran el conjunto SAM no habría cambios, por lo que se podría considerar un ítem para evaluar este motivo relacional con la pareja: "Para gustarle más a mi pareja".

\subsection{Validez de contenido de la escala a través del juicio de expertos}

Más del 80\% de los expertos señalaron que sí son adecuados los ítems del 3 al 5, 7 y 8 y del 10 al 12, del 14 al 16 correspondientes a motivos a favor de la reconstrucción mamaria, así como los ítems 17 y 18, del 20 al 22 y del 24 al 27, correspondientes a motivos contra la reconstrucción mamaria, por lo que estos 20 ítems fueron aceptados sin cambios. Debido a que ningún ítem fue señalado como inadecuado por al menos la mitad de los jueces, se conservaron los siete ítems restantes, y se procedió a modificar su redacción de acuerdo con las sugerencias dadas por los jueces (véase Tabla 5). 
Tabla 5. Porcentajes de adecuación de los ítems con base en el juicio de expertos

\begin{tabular}{|c|c|c|c|}
\hline ME HARÍA LA RECONSTRUCCIÓN MAMARIA & 1 & 2 & 3 \\
\hline 1. Porque me siento mal conmigo misma desde la mastectomía & 8,3 & 41,7 & 50 \\
\hline 2. Porque me siento menos atractiva & 8,3 & 16,7 & 75 \\
\hline 3. Porque me siento incompleta como mujer & 0 & 16,7 & 83,3 \\
\hline 4. Para mejorar mi autoestima & 0 & 0 & 100 \\
\hline 5. Porque no me gusta como se me ve ahora la ropa & 0 & 16,7 & 83,3 \\
\hline 6. Porque la prótesis externa me genera muchos problemas & 8,3 & 16,7 & 75 \\
\hline 7. Para mejorar mi estado emocional & 0 & 8,3 & 91,7 \\
\hline 8. Porque me daría mayor libertad de vestir todo tipo de ropa & 8,3 & 0 & 91,7 \\
\hline 9. Para verme como estaba antes de la mastectomía & 16,7 & 16,7 & 66,7 \\
\hline 10. Para sentirme más segura & 0 & 8,3 & 91,7 \\
\hline 11. Para verme simétrica de los pechos & 8,3 & 0 & 91,7 \\
\hline 12. Para sentirme más femenina & 8,3 & 8,3 & 83,3 \\
\hline 13. Para verme desnuda frente al espejo & $\mathbf{0}$ & 58,3 & 41,7 \\
\hline $\begin{array}{l}\text { 14. Para que mis hijos no vean algo raro o diferente en mis } \\
\text { pechos }\end{array}$ & 8,3 & $\mathbf{5 8 , 3}$ & 33,3 \\
\hline 15. Porque me permitiría cerrar este capítulo en mi vida & 8,3 & 8,3 & 83,3 \\
\hline 16. Para gustarle más a mi pareja & 16,7 & 0 & 83,3 \\
\hline \multicolumn{4}{|l|}{ NO ME HARÍA LA RECONSTRUCCIÓN MAMARIA } \\
\hline $\begin{array}{l}\text { 17. Porque tengo miedo de que se puedan presentar } \\
\text { complicaciones tras la reconstrucción }\end{array}$ & 0 & 16,7 & 83,3 \\
\hline 18. Porque me preocupa el alto costo que pueda tener & 0 & 16,7 & 83,3 \\
\hline 19. Porque estoy harta de tantos procedimientos quirúrgicos & 8,3 & 16,7 & 75 \\
\hline 20. Porque no es importante para mi & 0 & 0 & 100 \\
\hline 21. Porque me siento satisfecha con mi cuerpo tras la mastectomía & 0 & 8,3 & 91,7 \\
\hline 22. Porque a mi edad no la considero necesaria & 0 & 0 & 100 \\
\hline 23. Porque pienso que la recuperación ha de ser muy difícil & $\mathbf{0}$ & 33,3 & 66,7 \\
\hline 24. Porque me siento cómoda utilizando prótesis externa & 0 & 0 & 100 \\
\hline $\begin{array}{l}\text { 25. Porque tengo miedo del dolor que pueda sentir después de la } \\
\text { reconstrucción }\end{array}$ & 0 & 0 & 100 \\
\hline 26. Porque tengo miedo de que el cáncer regrese & 8,3 & 8,3 & 83,3 \\
\hline 27. Por mi estado de salud (diabetes, obesidad, hipertensión, etc.) & 8,3 & 8,3 & 83,3 \\
\hline
\end{tabular}

1 = No es adecuado, 2 = sí, pero requiere cambios, y 3 = sí, es adecuado.

Cinco de los doce expertos (41,7\%) señalaron que el ítem 1 ("porque me siento mal conmigo misma desde la mastectomía") requiere cambios, sugiriendo aclarar que si el malestar es de tipo emocional o físico. Tras revisar las respuestas de las pacientes se constató referencias a un malestar emocional, por lo que se modificó su redacción: "porque me siento mal emocionalmente desde la mastectomía". 
Dos de los doce expertos (16,7\%) indicaron que el ítem 2 ("porque me siento menos atractiva") requiere cambios, sugiriendo una redacción en sentido positivo o de mejora de la imagen corporal, por lo que se cambió a "para sentirme más atractiva".

Dos de los doce expertos $(16,7 \%)$ sugirieron cambiar la redacción del ítem 6 ("porque la prótesis externa me genera muchos problemas") en el sentido de darle un significado más específico, por ejemplo "porque la prótesis externa me es muy incómoda". No se siguió estas sugerencias y se mantuvo el sentido más amplio, ya que este ítem sintetiza asociaciones de distintos problemas con la prótesis externa.

También dos de los doce expertos (16,7\%) señalaron la necesidad de especificar si el ítem 9 ("para verme como estaba antes de la mastectomía") hace referencia a algo físico o emocional. Tras revisar las respuestas de las pacientes, se observó referencias a aspectos físicos, por lo que se cambió su redacción: "para verme físicamente como estaba antes de la mastectomía".

Siete de los 12 expertos (58,3\%) indicaron que el ítem 13 ("para verme desnuda frente al espejo") requiere ser modificado. La palabra "desnuda" fue señalada como inadecuada; no obstante, ninguno indicó otra opción de redacción. Cabe señalar que esta frase está tomada textualmente de las pacientes. Se cambió la redacción a: "para poder mirarme sin ninguna ropa frente al espejo".

Siete de los doce expertos $(58,3 \%)$ señalaron que el ítem 14 ("para que mis hijos no vean algo raro o diferente en mis pechos") necesita cambios, sugiriendo su redacción en positivo. Siguiendo esta sugerencia, el ítem se redactó del siguiente modo: "para que mis hijos vean mis pechos con apariencia más natural".

Dos de los doce expertos $(16,7 \%$ ) indicaron que el ítem 19 ("porque estoy harta de tantos procedimientos quirúrgicos") requiere cambios y sugirieron cambiar "harta" por "cansada". Se siguió la sugerencia.

Cuatro de los doce expertos $(33,3 \%)$ señalaron que el ítem 23 ("porque pienso que la recuperación ha de ser muy difícil") necesita cambios en su redacción, aclarando el significado de la dificultad. Tras revisar las respuestas de las pacientes, se observó que hacían referencia a dificultades en su vida diaria, por lo que se cambió la redacción: "porque pienso que durante la recuperación se me dificultará mucho realizar mis actividades diarias".

\section{Discusión}

Se formuló como objetivo describir los motivos para hacerse o no la reconstrucción mamaria a partir de la prueba de asociación libre acotada a un máximo de cinco palabras. En esta muestra de 65 mujeres con mastectomía unilateral, los motivos dados a favor de la reconstrucción mamaria se podría agrupar en cuatro tipos: estéticos o de imagen corporal ("por estética", "verse bien vestida", "simetría" "verse físicamente como estaba antes", "verse al espejo desnuda"), funcionales ("no tener problema con la prótesis externa", "facilidad para elegir ropa y vestirse"), emocionales o psicológicos ("sentirse bien consigo misma", "sentirse completa como mujer", "mejorar su autoestima", "mejorar su estado emocional", "sentirse más segura", "sentirse más femenina", "cerrar el ciclo del cáncer") y de relación ("por sus hijos", "por su pareja"). Cabe mencionar que por la pareja fue un motivo mencionado por cuatro mujeres y esta palabra definidora tuvo un valor semántico bajo, por lo que no 
quedó en el núcleo central. No obstante, si la ponderación se hiciese de 10 a 5, como se hace en otros estudios ${ }^{(21)}$, entonces quedaría en el núcleo central, pero el contenido de los hijos saldría del mismo. Considerando esta posibilidad y para completar los motivos relacionales, se redactó un ítem que evaluase el deseo de gustar o no perder atractivo ante la pareja, el cual quedó en la escala final sin necesidad de modificaciones tras el juicio de expertos.

En otros estudios se han encontrados motivos semejantes a los mencionados por este grupo de mujeres. En una muestra de 136 mujeres de Malasia con mastectomía, 37 optaron por la reconstrucción mamaria inmediata y señalaron como motivos: sentirse completa de nuevo, sentirse nuevamente femeninas, sentirse más equilibradas, ser libres para usar la ropa que quieran sin sentirse cohibidas, olvidarse del cáncer, evitar la prótesis externa, mejorar la relación marital y recomendación fuerte del cirujano ${ }^{(22)}$. En una muestra de 501 mujeres australianas con mastectomía, encontraron que el $94 \%$ de las 309 mujeres que se habían hecho reconstrucción mamaria reportaron como motivo querer verse y sentirse lo más normales posibles, $88 \%$ al considerarlo un paso en la recuperación completa del cáncer, $86 \%$ para mejorar su imagen corporal, $76 \%$ para que no estar acordándose todos los días del cáncer, $72 \%$ para sentirse a gusto al verse bien con la ropa y $68 \%$ por incomodidad con la prótesis ${ }^{(13)}$. En un estudio de revisión, se hallaron como contenidos temáticos más frecuentes a favor de la reconstrucción mamaria sentirse y verse normal; sentirse y verse bien; cuestiones prácticas en relación con la ropa y la próstesis; influencia de otros, como pareja, hijos o familia; y las expectativas de mejorar la relación y sexualidad de pareja, así como relaciones sociales y laborales ${ }^{(23)}$.

Los motivos en contra de la reconstrucción encontrados en el presente estudio podrían agrupar en cinco: miedos (miedo a complicaciones en la cirugía, miedo al dolor después de la cirugía, miedo a que regrese el cáncer), aceptación o comodidad con la situación presente ("me siento bien con mi cuerpo/aceptación", "no lo creo necesario/importante", "mayor seguridad/comodidad con prótesis externas"), costos ("por el costo/recurso económico", "dificultad para realizar su vida diaria en recuperación", "hartazgo por tantos procedimientos quirúrgicos") y salud ("por la edad ya no es conveniente/necesario", "sobrepeso"). Estos motivos también han sido encontrados en otros estudios previos. Entre las 99 mujeres malayas con mastectomía que no optaron por la reconstrucción mamaria inmediata, encontraron como sus principales razones: el miedo a una cirugía adicional, miedo a complicaciones en la cirugía, por no ser esencial para bienestar físico o emocional, edad avanzada y a que el cáncer regrese ${ }^{(22)}$. Entre las 192 mujeres australianas con mastectomía que no quisieron hacerse la reconstrucción mamaria, encontraron como motivos no querer más cirugías, no considerarlo necesario, no querer pasar por el proceso de recuperación, preocupación por las posibles complicaciones y efectos secundarios, no querer ponerse implantes y la falta de información ${ }^{(13)}$. En un estudio de revisión, destacó el miedo (al dolor, complicaciones, recurrencia y a quedar insatisfecha con el resultado), no querer más cirugía, no tener tiempo para someterse y recuperarse de una nueva cirugía, verlo como innecesario. También hallaron señalamientos de información inadecuada sobre la operación de reconstrucción, la falta de elección genuina para las mujeres y limitaciones adicionales de acceso debido a las barreras del sistema de salud ${ }^{(23)}$.

En el presente estudio los motivos en contra de la cirugía sobre falta de información, de opciones médicas y barreras del sistema sanitario reportados por Flitcroft et al. ${ }^{(23)}$ no fueron mencionadas. No obstante, se sugiere en futuros estudios indagar con 
preguntas directas sobre los mismos, ya que estas barreras son muy frecuentes en el sistema sanitario mexicano y pueden ser obviadas ${ }^{(24,25)}$. Precisamente, López et al. ${ }^{(12)}$ encontraron una marcada falta de información sobre el derecho a la opción de la reconstrucción mamaria sin costo para los derechohabientes del Instituto Mexicano del Seguro Social. Dos ítems para evaluar estos aspectos podrían ser: "me falta información sobre la reconstrucción mamaria como para tomar una decisión", y "desconozco las diversas opciones quirúrgicas de reconstrucción mamaria tras la mastectomía".

Desde los contenidos encontrados se redactaron 16 ítems sobre motivos para hacerse la reconstrucción y 11 en contra. Esta escala de 27 ítems fue evaluada en adecuación y comprensibilidad por un grupo de 12 expertos. Tras modificar la redacción de siete ítems la propuesta final de la escala conservó los 27 ítems iniciales. Finalmente, se han añadido dos más desde los argumentos finales sobre la falta de información, dando lugar a una escala de 29 ítems.

En conclusión, los contenidos asociados con los motivos para hacerse la reconstrucción mamaria con mayor frecuencia y valor semántico fueron 16, los cuales se pueden agrupar en estéticos o de imagen corporal, funcionales (prótesis externa, ropa y vestirse), emocionales o psicológicos y de relación con los hijos y la pareja. Los contenidos asociados en contra de hacerse la reconstrucción mamaria fueron 11 y pueden agruparse en miedos, aceptación o comodidad con la situación presente, costos y salud. Motivos sobre la falta de información, señalados en otros estudios, estuvieron ausentes en el presente estudio. Tras la redacción de 27 ítems a partir de estas asociaciones, con la modificación de 7 ítems por problemas de comprensibilidad o especificidad, y tras añadir dos ítems sobre el contenido ausente de falta de información, se alcanza la propuesta final de la escala con 29 ítems, finalizando la fase cualitativa del desarrollo del instrumento de medida.

Se sugiere estudiar la confiabilidad (consistencia interna y fiabilidad temporal) y validez (estructura dimensional, validez concurrente y predictiva) y distribución (baremos) de la escala de motivos a favor y en contra de la reconstrucción mamaria (EMRM). Una vez finalizada la fase cuantitativa que requeriría una muestra aleatoria grande, la escala estaría lista para su aplicación clínica e investigativa.

\section{Agradecimientos}

A Brizio Moreno, Paulina Herrera, Orestes Valles y Elisa Alejandra Garza Franco por su valioso apoyo en la aplicación del instrumento y el vaciado de datos.

\section{Referencias bibliográficas}

1. NIH consensus conference: Treatment of early-stage breast cancer. JAMA 1991;265:3915. doi:10.1001/jama.1991.03460030097037

2. Habermann EB, Abbott A, Parsons HM, Virnig BA, Al-Refaie WB, Tuttle TM. Are mastectomy rates really increasing in the United States? J Clin Oncol 2010;28:3437-41. doi:10.1200/JCO.2009.27.6774

3. Mohar A, Bargalló E, Ramírez MA, Lara F, Beltrán-Ortega A. Recursos disponibles para el tratamiento del cáncer de mama en México. Salud Publica Mex 2009;51:263-9. 
4. López-Carrillo L, Torres-Sánchez L, López-Cervantes M, Rueda-Neria C. Identificación de lesiones mamarias malignas en México. Salud Publica Mex 2001;43:199-202. doi:10.1590/S0036-36342001000300004

5. Ángeles-Llerenas A, Torres-Mejía G, Lazcano-Ponce E, Uscanga-Sánchez S, MaineroRatchelous F, Hernández-Ávila JE, Morales-Carmona E, Hernández-Ávila M. Efecto de la demora en la atención sobre la supervivencia de mujeres mexicanas con cáncer de mama. Salud Publica Mex 2016;58:237-50.

6. Tuttle TM, Habermann EB, Grund EH, Morris TJ, Virnig BA. Increasing use of contralateral prophylactic mastectomy for breast cancer patients: a trend toward more aggressive surgical treatment. J Clin Oncol 2007;25:5203-9. doi:10.1200/JCO.2007.12.3141

7. Morrow M, Harris JR. More mastectomies: is this what patients really want? J Clin Oncol 2009;27:4038-40. doi:10.1200/JCO.2009.23.0078

8. Rincón FMA, Pérez SGMA, Borda MM, Martín RG. Impacto de la reconstrucción mamaria sobre la autoestima y la imagen corporal en pacientes con cáncer de mama. Univ Psychol 2009;11:25-41.

9. Christian CK, Niland J, Edge SB, Ottesen RA, Hughes ME, Theriault R, Wilson J, Hergrueter CA, Weeks JC. A Multi-institutional analysis of the socioeconomic determinants of breast reconstruction. A study of the National Comprehensive Cancer Network. Ann Surg 2006;243:241-9. doi: 10.1097/01.sla.0000197738.63512.23

10. Greenberg CC, Lipsitz SR, Hughes ME, Edge SB, Theriault R, Wilson J, Carter WB, Blayney DW, Niland J, Weeks JC. Institutional variation in the surgical treatment of breast cancer: a study of the NCCN. Ann Surg 2011;254:339-45. doi:10.1097/ SLA.0b013e3182263bb0

11. Héquet D, Zarca K, Dolbeault S, Couturaud B, Ngô C, Fourchotte V, et al. Reasons of not having breast reconstruction: A historical cohort of 1937 breast cancer patients undergoing mastectomy. Springer plus 2013;2:325. doi:10.1186/2193-1801-2-325

12. López PI, Priego HR, Bracqbien CS, Ramos A. ¿Por qué las mujeres mastectomizadas no recurren a la reconstrucción mamaria en el sistema de seguridad social? Psicol Salud 2015;25:253-60.

13. Somogyi RB, Webb A, Baghdikian N, Stephenson J, Edward KL, Morrison W. Understanding the factors that influence breast reconstruction decision making in Australian women. Breast 2015;24:124-30. doi:10.1016/j.breast.2014.11.013

14. Yu KD, Di GH, Wu J, Lu JS, Shen KW, Shen ZZ, Shao ZM. Development and trends of surgical modalities for breast cancer in China: a review of 16-year data. Ann Surg Oncol 2007;14:2502-09. doi:10.1245/s10434-007-9436-2

15. Manne SL, Topham N, Kirstein L, Virtue SM, Brill K, Devine KA, Gajda T, Frederick S, Darabos K, Sorice K. Attitudes and decisional conflict regarding breast reconstruction among breast cancer patients. Cancer Nurs 2016;39:427-36. doi:10.1097/ ncc. 0000000000000320

16. Keith DJW, Walker MB, Walker LG, Heys SD, Sarkar TK, Hutcheon AW, Eremin O. Women who wish breast reconstruction: characteristics, fears and hopes. Plast Reconstr Surg 2003;111:1051-6. doi: 10.1097/01.PRS.0000046247.56810.40

17. Ananian P, Houvenaeghel G, Protière C, Rouanet P, Arnaud S, Moatti JP, Tallet A, Braud AC, Julian-Reynier C. Determinants of patients' choice of reconstruction with mastectomy for primary breast cancer. Ann Surg Oncol 2004;11:762-71. doi:10.1245/ASO.2004.11.027

18. Fu R, Chang MM, Chen M, Rohde C. A qualitative study of breast reconstruction decisionmaking among Asian immigrant women living in the United States. Plast Reconstr Surg 2017;139:360-8. doi:10.1097/PRS.0000000000002947 
19. Valdez JL. Las redes semánticas naturales, usos y aplicaciones en psicología social. Toluca de Lerdo: Universidad Autónoma del Estado de México, 2005.

20. Miaja M, Moral J. El significado psicológico de las cinco fases del duelo propuestas por Kübler-Ross mediante las redes semánticas naturales. Psicooncología 2013;10:109-30. doi:10.5209/rev_PSIC.2013.v10.41951

21. Hinojosa G. Ē tratamiento estadístico de las redes semánticas naturales. Revista Internacional de Ciencias Sociales y Humanidades 2008;18:133-54.

22. Shameem H, Yip CH, Fong E. Immediate breast reconstruction after mastectomy--why do women choose this option? Asian Pac J Cancer Prev 2008;9:409-12.

23. Flitcroft K, Brennan M, Spillane A. Making decisions about breast reconstruction: a systematic review of patients-reported factors influencing choice. Qual Life Res 2017;26:2287-319. doi:10.1007/s11136-017-1555-z.

24. Knaul FM, Nigenda G, Lozano R, Arreola-Ornelas H, Langer A, Frenk J. Cáncer de mama en México: una prioridad apremiante. Salud Publica Mex 2009;51:335-44.

25. Villarreal-Garza, C, Platas A, Martinez-Cannon BA, Bargalló-Rocha E, AguilarGonzález CN, Ortega-Leonard V, Ramos-Elías P, Hidalgo-Carrera J, Soto-Perez-de-Celis E. Information needs and internet use of breast cancer survivors in Mexico. Breast $\mathrm{J}$ 2016;23:373-5. doi: 10.1111/tbj.12747 


\section{ANEXO}

Tras la mastectomía, ¿qué tan de acuerdo está con los siguientes motivos para hacerse o no la reconstrucción mamaria? Por favor circula la opción de respuesta que mejor corresponda a lo que piensa o siente, contestando a las razones tanto para hacerse la reconstrucción (del 1 al 15) como para no hacérsela (del 16 al 26). Las respuestas varían de 1 = "totalmente en desacuerdo" a 7 "totalmente de acuerdo".

\begin{tabular}{|c|c|c|c|c|c|c|}
\hline 1 & 2 & 3 & 4 & 5 & 6 & 7 \\
\hline $\begin{array}{l}\text { Totalmente } \\
\text { en } \\
\text { desacuerdo }\end{array}$ & $\begin{array}{c}\text { Bastante } \\
\text { en } \\
\text { desacuerdo }\end{array}$ & $\begin{array}{c}\text { Algo } \\
\text { en } \\
\text { desacuerdo }\end{array}$ & $\begin{array}{c}\text { Ni de acuerdo } \\
\text { ni en } \\
\text { desacuerdo }\end{array}$ & $\begin{array}{l}\text { Algo } \\
\text { de } \\
\text { acuerdo }\end{array}$ & $\begin{array}{c}\text { Bastante } \\
\text { de } \\
\text { acuerdo }\end{array}$ & $\begin{array}{c}\text { Totalmente } \\
\text { de } \\
\text { acuerdo }\end{array}$ \\
\hline
\end{tabular}

\begin{tabular}{|l|l|l|l|l|l|l|l|}
\hline ME HARÍA LA RECONSTRUCCIÓN MAMARIA \\
\hline 1. Porque me siento mal emocionalmente desde la mastectomía & 1 & 2 & 3 & 4 & 5 & 6 & 7 \\
\hline 2. Para sentirme más atractiva & 1 & 2 & 3 & 4 & 5 & 6 & 7 \\
\hline 3. Porque me siento incompleta como mujer & 1 & 2 & 3 & 4 & 5 & 6 & 7 \\
\hline 4. Para mejorar mi autoestima & 1 & 2 & 3 & 4 & 5 & 6 & 7 \\
\hline 5. Porque no me gusta como se me ve ahora la ropa & 1 & 2 & 3 & 4 & 5 & 6 & 7 \\
\hline 6. Porque la prótesis externa me genera muchos problemas & 1 & 2 & 3 & 4 & 5 & 6 & 7 \\
\hline 7. Para mejorar mi estado emocional & 1 & 2 & 3 & 4 & 5 & 6 & 7 \\
\hline 8. Porque me daría mayor libertad de vestir todo tipo de ropa & 1 & 2 & 3 & 4 & 5 & 6 & 7 \\
\hline 9. Para verme físicamente como estaba antes de la mastectomía & 1 & 2 & 3 & 4 & 5 & 6 & 7 \\
\hline 10. Para sentirme más segura & 1 & 2 & 3 & 4 & 5 & 6 & 7 \\
\hline 11. Para verme simétrica de los pechos & 1 & 2 & 3 & 4 & 5 & 6 & 7 \\
\hline 12. Para sentirme más femenina & 1 & 2 & 3 & 4 & 5 & 6 & 7 \\
\hline 13. Para poder mirarme sin ninguna ropa frente al espejo & 1 & 2 & 3 & 4 & 5 & 6 & 7 \\
\hline 14. Para que mis hijos vean mis pechos con apariencia más natural & 1 & 2 & 3 & 4 & 5 & 6 & 7 \\
\hline 15. Porque me permitiría cerrar este capítulo en mi vida & 1 & 2 & 3 & 4 & 5 & 6 & 7 \\
\hline 16. Para gustarle más a mi pareja & 1 & 2 & 3 & 4 & 5 & 6 & 7 \\
\hline
\end{tabular}

\section{NO ME HARÍA LA RECONSTRUCCIÓN MAMARIA}

\begin{tabular}{|l|l|l|l|l|l|l|l|}
\hline $\begin{array}{l}\text { 17. Porque tengo miedo de que se puedan presentar complicaciones } \\
\text { tras la reconstrucción }\end{array}$ & 1 & 2 & 3 & 4 & 5 & 6 & 7 \\
\hline 18. Porque me preocupa el alto costo económico que pueda tener & 1 & 2 & 3 & 4 & 5 & 6 & 7 \\
\hline 19. Porque estoy cansada de tantos procedimientos quirúrgicos & 1 & 2 & 3 & 4 & 5 & 6 & 7 \\
\hline 20. Porque no es importante para mi & 1 & 2 & 3 & 4 & 5 & 6 & 7 \\
\hline 21. Porque me siento satisfecha con mi cuerpo tras la mastectomía & 1 & 2 & 3 & 4 & 5 & 6 & 7 \\
\hline 22. Porque a mi edad no la considero necesaria & 1 & 2 & 3 & 4 & 5 & 6 & 7 \\
\hline $\begin{array}{l}\text { 23. Porque pienso que durante la recuperación se me dificultará } \\
\text { mucho realizar mis actividades diarias }\end{array}$ & 1 & 2 & 3 & 4 & 5 & 6 & 7 \\
\hline 24. Porque me siento cómoda utilizando prótesis externa & 1 & 2 & 3 & 4 & 5 & 6 & 7 \\
\hline $\begin{array}{l}\text { 25. Porque tengo miedo del dolor que pueda sentir después de la } \\
\text { reconstrucción }\end{array}$ & 1 & 2 & 3 & 4 & 5 & 6 & 7 \\
\hline 26. Porque tengo miedo de que el cáncer regrese & 1 & 2 & 3 & 4 & 5 & 6 & 7 \\
\hline 27. Por mi estado de salud (diabetes, obesidad, hipertensión, etc.) & 1 & 2 & 3 & 4 & 5 & 6 & 7 \\
\hline $\begin{array}{l}\text { 28. Me falta información sobre la reconstrucción mamaria como } \\
\text { para tomar una decisión }\end{array}$ & 1 & 2 & 3 & 4 & 5 & 6 & 7 \\
\hline $\begin{array}{l}\text { 29. Desconozco las diversas opciones quirúrgicas de reconstrucción } \\
\text { mamaria tras la mastectomía }\end{array}$ & 1 & 2 & 3 & 4 & 5 & 6 & 7 \\
\hline
\end{tabular}

